\title{
High-Field Approximations of the Energy-Transport Model for Semiconductors with Non-Parabolic Band Structure
}

Pierre Degond

Ansgar Jüngel

Konstanzer Schriften in Mathematik und Informatik

Nr. 126, August 2000

ISSN 1430-3558

Konstanzer Online-Publikations-System (KOPS)

URL: http://www.ub.uni-konstanz.de/kops/volltexte/2006/2096/

(C) Fachbereich Mathematik und Statistik

(c) Fachbereich Informatik und Informationswissenschaft

Universität Konstanz

Fach D 188, 78457 Konstanz, Germany

Email: preprints@informatik.uni-konstanz.de

WWW: http://www.informatik.uni-konstanz.de/Schriften/ 


\title{
High-Field Approximations of the \\ ENERGY-TRANSPORT MODEL FOR SEMICONDUCTORS \\ with Non-Parabolic Band Structure
}

\author{
Pierre Degond \\ Laboratoire MIP, Université Paul Sabatier, \\ 118 route de Narbonne, 31062 Toulouse Cedex, France, \\ e-mail: degond@mip.ups-tlse.fr

\section{Ansgar Jüngel} \\ Fachbereich Mathematik und Statistik, Universität Konstanz, \\ Fach D193, 78457 Konstanz, Germany, \\ e-mail: juengel@fmi . uni-konstanz.de
}

\begin{abstract}
An asymptotic analysis of the energy-transport equations for semiconductors with the scaled energy relaxation time as small parameter is performed. Using a variant of the Chapman-Enskog method, high-field drift-diffusion models are derived. Furthermore, the dependence of the macroscopic parameters such as the diffusivity are investigated for parabolic and non-parabolic band approximations (in the sense of Kane). Explicit expressions of the physical parameters are obtained.
\end{abstract}

Keywords. High-field drift-diffusion models, Chapman-Enskog method, nonparabolic band, semiconductors.

Acknowledgements. The authors acknowledge partial support of the TMR network "Asymptotic methods in kinetic theory", grant no. ERB-FMBX-CT970157 and of the DAAD-Procope Program. The second author was supported by the Gerhard-Hess Program of the Deutsche Forschungsgemeinschaft, grant no. JU359/3-1. 


\section{Introduction}

The accurate modeling of high-field effects is an important task in the simulation of charge carrier flow in modern semiconductor devices. A standard and commonly used approach is the inclusion of field-dependent transport parameters in macroscopic models. Often field-dependent mobilities are employed in standard drift-diffusion models [15]. The detailed form of the field dependence is then obtained by heuristic arguments combined with fitting to experimental values or to results of Monte-Carlo simulations of the semiconductor Boltzmann equation $[9]$.

Recently, also more rigorous approaches have been considered. A drift-diffusion model with field-dependent mobility has been derived by Poupaud in [13]. There the drift-diffusion model is a result of a two-step procedure. First, by a limit process starting from the semiconductor Boltzmann equation, an equation without diffusion term is derived. Then the diffusion term with a field-dependent diffusion constant is obtained as a higher-order correction by a variant of the Chapman-Enskop method (arrow 1 in Fig. 1). However, this result has the disadvantage that the assumptions on the scattering mechanisms are physically unrealistic.

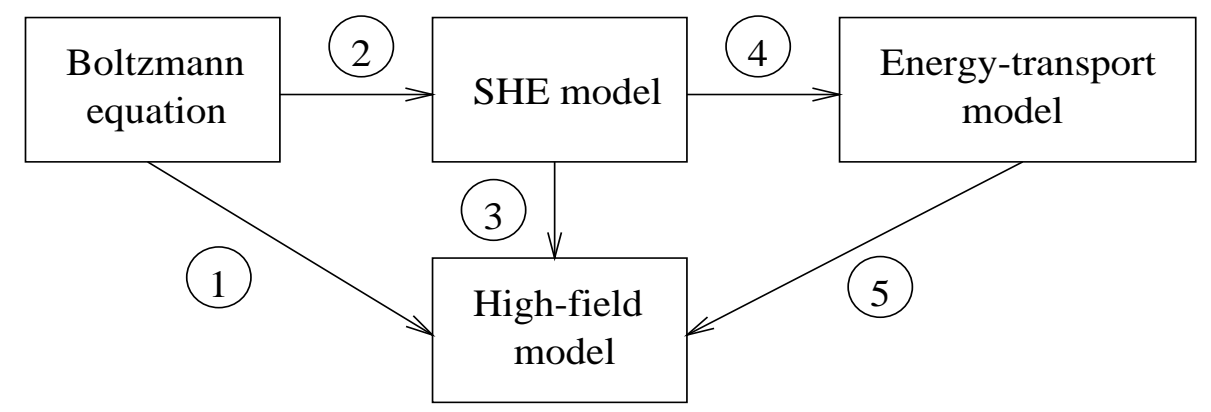

Figure 1: Derivation of high-field models.

Another approach has been employed by Ben Abdallah et al. [3]. They derive high-field models from the Spherical Harmonics Expansion (SHE) model. The SHE model can be obtained either by expansion of the distribution function in terms of spherical harmonics [16, 17], or by a Hilbert expansion assuming that elastic collisions are the dominating physical effect $[1,8,14]$. The latter approach seems more attractive since it can be easily applied to non-rotationally symmetric band structures (arrow 2). In a second limit with dominating inelastic scattering, Ben Abdallah et al. derive high-field drift-diffusion models (arrow 3). However, in this approach an operator equation (containing the collision operator) has to be inverted, and thus the field dependence is not always explicit.

In this paper we will derive high-field drift-diffusion equations with explicit field-dependent diffusivities. We derive these models from the energy-transport 
equations. The energy-transport equations can be obtained from the Boltzmann equation assuming dominating inelastic scattering mechanisms [2] or from the SHE model assuming dominant electron-electron collisions [1] (arrow 4). The advantage of the energy-transport model is that explicit expressions for the diffusion coefficients can be given even for non-parabolic band diagrams [7].

In Section 2 the energy-transport model is presented, the physical assumptions on the band structure and the relaxation mechanism are given, and a high-field scaling is introduced. The limiting equation is a (first order) convection equation for the macroscopic electron density with field-dependent mobility.

For the non-parabolic band approximation in the sense of Kane [11], we derive in Section 3 explicit expressions for the electron mean velocity and electron temperature. In the parabolic band approximation, we obtain the same fielddependent mobilities as in the literature.

By a variant of the Chapman-Enskog method, a (second order) correction is constructed in Section 4 which yields a high-field drift-diffusion equation (arrow 5). For general band diagrams, this model is of the form

$$
\begin{aligned}
\partial_{t} n-\operatorname{div}(D(n, E) \nabla n+G(n, E)) & =0, \\
\operatorname{div} E & =n-C(x),
\end{aligned}
$$

where $n$ is the electron density, $E$ is the electric field, and $C(x)$ is the doping concentration characterizing the semiconductor device. The diffusion matrix $D(n, E)$ and the drift term $G(n, E)$ are given explicitely. We prove that the matrix $D(n, E)$ is positive definite for parabolic and non-parabolic band diagrams, i.e. the above equation for $n$ is parabolic.

For parabolic bands in the Chen case [5] the above equation for $n$ simplifies:

$$
\partial_{t} n-\operatorname{div}\left(d(|E|) \nabla n+\frac{n}{T(|E|)} E\right)=0
$$

where the diffusivity $d(|E|)$ and the temperature $T(|E|)$ are given by

$$
d(|E|)=\frac{d_{0}}{2-T_{0} / T(|E|)}, \quad T(|E|)=\frac{1}{2}\left(T_{0}+\sqrt{T_{0}^{2}+\frac{8}{3} d_{0} \tau_{0}|E|^{2}}\right),
$$

$d_{0}, \tau_{0}$ are diffusivity and relaxation time constants, respectively, defined in (3.6), and $T_{0}=1$ is the (scaled) ambient temperature. In the limit $|E| \rightarrow \infty$ the mobility $d(|E|)$ converges to $d_{0} / 2$ which is half of the low-field diffusivity $d_{0}$.

We stress once more the fact that compared to the paper [3], we derive $e x$ plicit expressions for the coefficients in the drift-diffusion equation. Therefore, the proposed models can be solved numerically (using, for instance, the methods in $[4,7])$ and the results can be compared to Monte-Carlo simulations of the Boltzmann equation. The numerical simulation will be performed in a forthcoming publication. 


\section{Assumptions - scaling}

Consider the dimensionless energy-transport equations in the entropy variables $\mu / T$ and $-1 / T[1]$ :

$$
\begin{aligned}
\partial_{t} n+\operatorname{div} j_{n} & =0 \\
\partial_{t} W+\operatorname{div} j_{W} & =-E \cdot j_{n}+R, \\
j_{n} & =-L_{11}\left(\nabla \frac{\mu}{T}+\frac{E}{T}\right)-L_{12} \nabla\left(-\frac{1}{T}\right), \\
j_{W} & =-L_{21}\left(\nabla \frac{\mu}{T}+\frac{E}{T}\right)-L_{22} \nabla\left(-\frac{1}{T}\right), \\
\lambda^{2} \operatorname{div} E & =n-C(x) .
\end{aligned}
$$

These equations are solved in a bounded domain of $\mathbb{R}^{N}(N \geq 1)$ and are supplemented with appropriate initial and boundary conditions (see [10]). The physical variables are the chemical potential $\mu$, the electron temperature $T$, and the electric field $E=-\nabla \phi$ with the electrostatic potential $\phi$. The electron density $n$, the density of the internal energy $W$, the energy relaxation term $R$ and the diffusion coefficients $L_{i j}$ depend on the entropy variables $\mu / T$ and $-1 / T$. Furthermore, $j_{n}$ and $j_{W}$ are the particle and energy current densities, $\lambda>0$ the scaled Debye length, and $C(x)$ the doping profile characterizing the device under consideration.

The existence of weak solutions of the system (2.1)-(2.5) with mixed DirichletNeumann boundary conditions and initial conditions for the variables has been shown in [6] under some assumptions on the nonlinear functions. The equations have been numerically solved in, e.g., [7].

We impose the following physical assumptions:

(H1) The energy-band diagram $\varepsilon$ of the semiconductor crystal is spherically symmetric, differentiable and a strictly monotone function of the modulus $k=|\vec{k}|$ of the wave vector $\vec{k}$. Therefore, the Brillouin zone equals $\mathbb{R}^{3}$ and $\varepsilon: \mathbb{R} \rightarrow \mathbb{R}, \quad k \mapsto \varepsilon(k)$.

(H2) The momentum relaxation time is given by

$$
\tau(\varepsilon)=\left(\phi_{0}\left(2 N_{0}+1\right) \varepsilon^{\beta} N(\varepsilon)\right)^{-1}, \quad \beta \geq 0, \quad \phi_{0}>0,
$$

where $N(\varepsilon)=4 \pi k^{2} /\left|\varepsilon^{\prime}(k)\right|$ is the density of states of energy $\varepsilon=\varepsilon(k)[1$, (III.31)], $\phi_{0}$ denotes the transition matrix constant, and $N_{0}$ is the phonon occupation number [3, Sect.4].

(H3) The electron density $n$ and the internal energy $W$ are given by non-degenerate Boltzmann statistics. 
(H4) The energy relaxation is given by [1, (IV.18)]

$$
R=\int_{0}^{\infty} S_{1}\left(e^{(\mu-\varepsilon) / T}\right) \varepsilon d \varepsilon
$$

and the phonon collision operator $S_{1}$ reads in the Fokker-Planck approximation

$$
S_{1}\left(e^{(\mu-\varepsilon) / T}\right)=\frac{\partial}{\partial \varepsilon}\left\{\phi_{0} \varepsilon^{\beta} N(\varepsilon)^{2}\left[\left(1+T_{0} \frac{\partial}{\partial \varepsilon}\right) e^{(\mu-\varepsilon) / T}\right]\right\},
$$

where $T_{0}=1$ is the (scaled) ambient temperature.

Under the hypothese (H1)-(H3), the diffusion coefficients are given by

$$
L_{i j}=e^{\mu / T} \int_{0}^{\infty} \lambda_{0}(\varepsilon) \varepsilon^{i+j-2} e^{-\varepsilon / T} d \varepsilon
$$

where

$$
\lambda_{0}(\varepsilon)=\frac{4 \pi}{3} \tau(\varepsilon)\left|\varepsilon^{\prime}(k)\right| k^{2} \quad \text { and } \quad \varepsilon=\varepsilon(k)
$$

(see [1, (IV.17)(III.33)]). We refer to [1] for more general expressions for the diffusion coefficients under weaker assumptions. From assumption (H3), we get for the electron density and internal energy the expressions [1, (IV.16)]

$$
\begin{aligned}
n & =e^{\mu / T} \int_{0}^{\infty} e^{-\varepsilon / T} N(\varepsilon) d \varepsilon \\
W & =e^{\mu / T} \int_{0}^{\infty} e^{-\varepsilon / T} N(\varepsilon) \varepsilon d \varepsilon .
\end{aligned}
$$

The expression for the energy relaxation term can be simplified using (H4):

$$
R=\phi_{0} e^{\mu / T}\left(T_{0}-T\right) \int_{0}^{\infty} \varepsilon^{\beta} N(\varepsilon)^{2} e^{-\varepsilon / T} d \varepsilon
$$

Let $\gamma(\varepsilon)=k^{2}$ be the inverted $\varepsilon(k)$ relation. Then we can write the diffusion coefficients, the electron density, the internal energy and the energy relaxation term as follows (see [7] for details):

$$
\begin{aligned}
L_{i j} & =e^{\mu / T} T^{i+j-\beta-1} P_{\beta}^{i+j}(T), \\
n & =e^{\mu / T} T Q^{0}(T) \\
W & =e^{\mu / T} T^{2} Q^{1}(T) \\
R & =e^{\mu / T} T^{\beta} R_{\beta}(T)\left(T_{0}-T\right),
\end{aligned}
$$


and the functions $P_{\beta}^{k}, Q^{l}$ and $R_{\beta}$ are defined by

$$
\begin{aligned}
P_{\beta}^{k}(T) & =\frac{4}{3 \phi_{o}\left(2 N_{0}+1\right)} \int_{0}^{\infty} \frac{\gamma(T u)}{\gamma^{\prime}(T u)^{2}} u^{k-\beta-2} e^{-u} d u \\
Q^{l}(T) & =2 \pi \int_{0}^{\infty} \gamma(T u)^{1 / 2} \gamma^{\prime}(T u) u^{l} e^{-u} d u \\
R_{\beta}(T) & =4 \pi^{2} \phi_{0} \int_{0}^{\infty} \gamma(T u) \gamma^{\prime}(T u)^{2} u^{\beta} e^{-u} d u
\end{aligned}
$$

where $k=2,3,4$ and $l=0,1$.

We introduce now the scaling

$$
x \rightarrow \delta x, \quad t \rightarrow \delta t, \quad \phi \rightarrow \frac{1}{\delta} \phi
$$

for "small" $\delta>0$. This scaling implies that the electric field $-\nabla \phi$ remains unchanged, which means that we assume the potential to have variations of order 1 over the microscopic scale. Then the rescaled equations (2.1)-(2.5) read

$$
\begin{aligned}
\partial_{t} n+\operatorname{div} j_{n} & =0 \\
\partial_{t} W+\operatorname{div} j_{W} & =\frac{1}{\delta}\left(-E \cdot j_{n}+R\right), \\
j_{n} & =-L_{11}\left(\delta \nabla \frac{\mu}{T}+\frac{E}{T}\right)-\delta L_{12} \nabla\left(-\frac{1}{T}\right), \\
j_{W} & =-L_{21}\left(\delta \nabla \frac{\mu}{T}+\frac{E}{T}\right)-\delta L_{22} \nabla\left(-\frac{1}{T}\right), \\
\delta \lambda^{2} \operatorname{div} E & =n-C(x) .
\end{aligned}
$$

Assuming that in the limit $\delta \rightarrow 0, \delta \lambda^{2}$ is of order $O(1)$, we obtain (formally) the limit equations,

$$
\begin{gathered}
\partial_{t} n+\operatorname{div} j_{n}=0, \quad-E \cdot j_{n}+R=0 \\
j_{n}=-L_{11} \frac{E}{T}, \quad j_{w}=-L_{21} \frac{E}{T}, \quad \operatorname{div} E=n-C(x) .
\end{gathered}
$$

The second equation in (2.19) and the first equation in (2.20) imply that for given electric field $E$, the electron temperature $T$ can be computed according to

$$
|E|^{2}=-T \frac{R(\mu / T,-1 / T)}{L_{11}(\mu / T,-1 / T)}
$$

Notice that for $E=0, T=T_{0}$ solves $(2.21)$ since $R=0$ at $T=T_{0}$. We suppose that the nonlinear equation (2.21) for $T$ can be solved uniquely:

(H5) For all $|E| \geq 0$ and $\mu \in \mathbb{R}$, there exists a unique solution $T=T(\mu,|E|)$ of $(2.21)$. 
If we only assume solvability of Eq. (2.21), this equation may have more than one solution $T$. The corresponding bifurcation problem will be studied in a future publication.

In view of the relations (2.7) and (2.10) for $L_{11}$ and $R$, Eq. (2.21) can be reformulated as

$$
|E|^{2}=T^{2 \beta}\left(T-T_{0}\right) \frac{R_{\beta}(T)}{P_{\beta}^{2}(T)}=: f(T),
$$

i.e. in fact, $T$ only depends on $|E|$ (and not on $\mu$ ). A sufficient condition for (H5) is for instance the following assumption on $f$ which is in fact an assumption on the energy band structure given by $\gamma$ :

(H6) $f$ is strictly increasing on $\left[T_{0}, \infty\right)$ and $\lim _{T \rightarrow \infty} f(T)=\infty$.

We show below that for parabolic bands and non-parabolic bands in the sense of Kane, the property (H6) on $f$ is satisfied (see Lemma 3.1).

Let $f$ be differentiable and let (H6) hold. Then the function $|E| \mapsto T(|E|)$ has the following properties:

$$
T(0)=T_{0}, \quad T^{\prime}(|E|)>0 \text { for all }|E|>0, \quad \lim _{|E| \rightarrow \infty} T(|E|)=\infty .
$$

We are also interested in the qualitive behavior of the mean electron velocity $v_{n}$ depending on $|E|$. The velocity $v_{n}$ is defined by

$$
j_{n}=-n v_{n}
$$

which gives from (2.20) the expression

$$
v_{n}=\frac{L_{11}}{n} \frac{E}{T},
$$

or with (2.7) and (2.8):

$$
v_{n}=v_{n}(E)=T(|E|)^{-1-\beta} \frac{P_{\beta}^{2}(T(|E|))}{Q^{0}(T(|E|))} E .
$$

Then the mobility $\mu_{n}$ is given by $v_{n}(|E|)=\mu_{n}(|E|) E$. Without specifying the band structure, it is difficult to describe the qualitative behavior of the function $E \mapsto v_{n}(E)$. In particular, the limit $\left|v_{n}(E)\right|$ as $|E| \rightarrow \infty$ depends strongly on the assumptions on the band structure. In the general case, we can only conclude that

$$
\left|v_{n}(E)\right|>0 \text { for }|E|>0 \quad \text { and } \quad v_{n}(0)=0
$$




\section{High-field drift models}

The high-field drift model for general band structure (assuming (H1)-(H5)) is given by Eqs. (2.19) and (2.23):

$$
\begin{aligned}
\partial_{t} n-\operatorname{div}\left(n v_{n}(E)\right) & =0 \\
v_{n}(E) & =T(|E|)^{-1-\beta} \frac{P_{\beta}^{2}(T(|E|))}{Q^{0}(T(|E|))} E, \\
\operatorname{div} E & =n-C(x),
\end{aligned}
$$

and $T(|E|)$ is defined as the unique solution of $|E|^{2}=f(T)$ (see (2.22)). In this section we will derive explicit expressions for $v_{n}(E)$ and $T(|E|)$ for more specific band diagrams, in particular for non-parabolic bands in the sense of Kane and for parabolic bands.

\subsection{Non-parabolic band approximation}

The non-parabolic band structure in the sense of Kane [11] is defined as follows:

(H7) Let the energy $\varepsilon(k)$ satisfy

$$
\varepsilon(1+\alpha \varepsilon)=\frac{k^{2}}{2 m^{*}}, \quad \alpha>0 .
$$

The constant $m^{*}$ is the (scaled) effective electron mass given by $m^{*}=m_{0} k_{B} T_{0} /$ $\hbar^{2} k_{0}^{2}$, where $m_{0}$ is the unscaled effective mass, $k_{B}$ is the Boltzmann constant, $\hbar$ is the reduced Planck constant, $k_{0}$ is a typical wave vector, and $\alpha>0$ is the (scaled) non-parabolicity parameter. When $\alpha=0$, we get a parabolic band structure.

Under the assumption (H7), Eqs. (2.22) and (2.23) become

$$
\begin{aligned}
|E|^{2} & =\left(d_{0} \tau_{0}\right)^{-1} T^{2 \beta}\left(T-T_{0}\right) \frac{r_{\beta}(\alpha T)}{P_{\beta}(\alpha T)}=f(T), \\
v_{n}(E) & =d_{0} T^{-1 / 2-\beta} \frac{P_{\beta}(\alpha T)}{q(\alpha T)} E,
\end{aligned}
$$

where

$$
\tau_{0}=\left(2 \pi \phi_{0}\left(2 m^{*}\right)^{3 / 2}\right)^{-1}, \quad d_{0}=\left(3 \pi \phi_{0}\left(2 N_{0}+1\right) m^{*}\left(2 m^{*}\right)^{3 / 2}\right)^{-1 / 2}
$$

and

$$
\begin{aligned}
P_{\beta}(\alpha T) & =\int_{0}^{\infty} \frac{1+\alpha T u}{(1+2 \alpha T u)^{2}} u^{1-\beta} e^{-u} d u, \\
q(\alpha T) & =\int_{0}^{\infty}(1+\alpha T u)^{1 / 2}(1+2 \alpha T u) u^{1 / 2} e^{-u} d u, \\
r_{\beta}(\alpha T) & =\Gamma(2+\beta)+5 \Gamma(3+\beta) \alpha T+8 \Gamma(4+\beta)(\alpha T)^{2}+4 \Gamma(5+\beta)(\alpha T)^{3},
\end{aligned}
$$


and $\Gamma$ is the Gamma function defined by

$$
\Gamma(s)=\int_{0}^{\infty} u^{s-1} e^{-u} d u \quad \text { for } s>0 .
$$

These formulas follow from Eqs. (2.11)-(2.13) and $\gamma(T u)=2 m^{*} T u(1+\alpha T u)$.

First we show that Hypothesis (H5) is satisfied for non-parabolic and parabolic band approximations:

Lemma 3.1 Let (H1)-(H4) and (H7) for $\alpha \geq 0$ hold and let $\beta<2$. Then for any $|E| \geq 0, E q$. (3.4) has a unique solution $T=T(|E|)$, i.e. Hypothesis (H5) is satisfied.

Proof. We show that the function $f$ defined in (2.22) satisfies (H6). Since

$$
P_{\beta}(\alpha T) \leq \int_{0}^{\infty}(1+\alpha T u) u^{1-\beta} e^{-u} d u=\Gamma(2-\beta)+\Gamma(3-\beta) \alpha T
$$

(here we need the condition $\beta<2$ ), we get

$$
f(T) \geq\left(d_{0} \tau_{0}\right)^{-1} T^{2 \beta}\left(T-T_{0}\right) \frac{r_{\beta}(\alpha T)}{\Gamma(2-\beta)+\Gamma(3-\beta) \alpha T} .
$$

The term $r_{\beta}(\alpha T)$ is of order $O\left(T^{3}\right)$ as $T \rightarrow \infty$, therefore $f(T) \rightarrow \infty$ as $T \rightarrow \infty$.

In order to show that $f$ is strictly increasing, it is sufficient to prove that both $T \mapsto T^{2 \beta}\left(T-T_{0}\right)$ and $T \mapsto r_{\beta}(\alpha T) / P_{\beta}(\alpha T)$ are strictly increasing. For the first function, this is clear. For the second function we compute its derivative:

$$
\frac{d}{d T} \frac{r_{\beta}(\alpha T)}{P_{\beta}(\alpha T)}=\frac{\alpha}{P_{\beta}(\alpha T)^{2}}\left(r_{\beta}^{\prime}(\alpha T) P_{\beta}(\alpha T)-r_{\beta}(\alpha T) P_{\beta}^{\prime}(\alpha T)\right)>0
$$

since $r_{\beta}^{\prime}(\alpha T) \geq 5 \Gamma(3+\beta)>0$ and

$$
P_{\beta}^{\prime}(\alpha T)=-\int_{0}^{\infty} \frac{3+2 \alpha T u}{(1+2 \alpha T u)^{3}} u^{2-\beta} e^{-u} d u<0 .
$$

Hence, $f$ satisfies (H6) and therefore (H5).

Lemma 3.2 Let (H1)-(H4) and (H7) hold. Then, as $|E| \rightarrow \infty$,

$$
T(|E|)=O\left(|E|^{2 /(5+2 \beta)}\right), \quad\left|v_{n}(E)\right|=O\left(|E|^{-1 /(5+2 \beta)}\right) .
$$

Proof. The definitions of $P_{\beta}(\alpha T), r_{\beta}(\alpha T)$ and $q(\alpha T)$ imply

$$
P_{\beta}(\alpha T)=O\left(T^{-1}\right), \quad r_{\beta}(\alpha T)=O\left(T^{3}\right), \quad q(\alpha T)=O\left(T^{-3 / 2}\right) \quad \text { as } T \rightarrow \infty,
$$


and hence, from (3.4),

$$
|E|^{2}=O\left(T^{5+2 \beta}\right) \quad \text { as } T \rightarrow \infty .
$$

Since $|E| \rightarrow \infty$ implies $T \rightarrow \infty$, we get

$$
T=O\left(|E|^{2 /(5+2 \beta)}\right) \quad \text { as }|E| \rightarrow \infty .
$$

Furthermore, by (3.5),

$$
\left|v_{n}(E)\right|=\sqrt{\frac{d_{0}}{\tau_{0}}\left(1-\frac{T_{0}}{T}\right)} \frac{\sqrt{P_{\beta}(\alpha T) r_{\beta}(\alpha T)}}{q(\alpha T)}=O\left(T^{-1 / 2}\right) \quad \text { as } T \rightarrow \infty,
$$

and

$$
\left|v_{n}(E)\right|=O\left(|E|^{-1 /(5+2 \beta)}\right) \quad \text { as }|E| \rightarrow \infty .
$$

The lemma is proved.

Remark 3.3 The mean velocity derived from the SHE model in the mean-free path limit for the cases $\beta=0$ and $\beta=\frac{1}{2}$ has the same qualitative behavior as above (see [3, Ex. 4.3 and 4.4]).

\subsection{Parabolic band approximation}

In the parabolic band case $\varepsilon(k)=k^{2} / 2 m^{*}$ for $\beta=0$ and $\beta=\frac{1}{2}$, we can give explicit formulas for $T(|E|)$ and $v_{n}(E)$.

Lemma 3.4 Let (H1)-(H4) hold and let $\varepsilon=k^{2} / 2 m^{*}$ and $\beta<2$. Then $T=$ $T(|E|)$ is unique solution of the nonlinear equation

$$
|E|^{2}=\frac{\Gamma(2+\beta)}{d_{0} \tau_{0} \Gamma(2-\beta)} T^{2 \beta}\left(T-T_{0}\right)
$$

and

$$
v_{n}(E)=\sqrt{\frac{d_{0}}{\tau_{0}} \Gamma(2-\beta) \Gamma(2+\beta)\left(1-\frac{T_{0}}{T(|E|)}\right)} \frac{E}{|E|} .
$$

The velocity satisfies, as $|E| \rightarrow \infty$,

$$
\left|v_{n}(E)\right| \rightarrow v_{\infty}:=\frac{2}{\sqrt{\pi}} \sqrt{\Gamma(2-\beta) \Gamma(2+\beta)} \sqrt{\frac{d_{0}}{\tau_{0}}} .
$$

Moreover, in the Chen case $\beta=\frac{1}{2}$ [5], it holds

$$
\begin{aligned}
T(|E|) & =\frac{1}{2}\left(T_{0}+\sqrt{T_{0}^{2}+\frac{8}{3} d_{0} \tau_{0}|E|^{2}}\right), \\
v_{n}(E) & =\frac{2 d_{0} E}{T_{0}+\sqrt{T_{0}^{2}+\frac{8}{3} d_{0} \tau_{0}|E|^{2}}}
\end{aligned}
$$


with $v_{\infty}=\sqrt{3 d_{0} / 2 \tau_{0}}$, and in the Lyumkis case $\beta=0$ [12] we have

$$
\begin{aligned}
T(|E|) & =T_{0}+\frac{1}{C_{T}}|E|^{2}, \\
v_{n}(E) & =\frac{4 d_{0}}{\sqrt{\pi}} \frac{E}{\sqrt{T_{0}+d_{0} \tau_{0}|E|^{2}}}
\end{aligned}
$$

with $v_{\infty}=4 \sqrt{d_{0} / \pi \tau_{0}}$.

Proof. Eqs. (3.8) and (3.9) follow from (3.4) and (3.7) since $r_{\beta}(0)=\Gamma(2+$ $\beta), P_{\beta}(0)=\Gamma(2-\beta)$ and $q(0)=\Gamma(3 / 2)=\sqrt{\pi} / 2$. Furthermore,

$$
\left|v_{n}(E)\right|^{2}=\frac{4}{\pi} \frac{d_{0}}{\tau_{0}} \Gamma(2-\beta) \Gamma(2+\beta)\left(1-\frac{T_{0}}{T}\right) \rightarrow v_{\infty}^{2}
$$

as $|E| \rightarrow \infty$ or, equivalently, $T \rightarrow \infty$. In the Chen case $\beta=\frac{1}{2}$ we have to solve the equation

$$
|E|^{2}=\frac{3}{2 d_{0} \tau_{0}} T\left(T-T_{0}\right)
$$

(using $\Gamma(5 / 2)=3 \sqrt{\pi} / 4$ ), which gives (3.10) and (3.11). Finally, if $\beta=0$, we have to solve

$$
|E|^{2}=\frac{1}{d_{0} \tau_{0}}\left(T-T_{0}\right),
$$

from which we immediately conclude (3.12) and (3.13).

Remark 3.5 Notice that we have velocity saturation both in the parabolic and non-parabolic case (see Lemma 3.2). In the non-parabolic case however, the limit velocity vanishes. This behavior has already been observed in [3].

\section{High-field drift-diffusion models}

In Section 3 we have derived high-field models with no diffusion terms. Diffusion can be obtained as a higher-order correction by using a variant of the ChapmanEnskog method.

\subsection{General band structure}

Let the assumptions (H1)-(H5) hold and let $\left(n^{\delta}, T^{\delta}\right)$ be a solution to (2.14) (2.17), defining the chemical potential $\mu$ and the internal energy $W$ (uniquely) via

$$
\mu^{\delta}=\mu\left(n^{\delta}, T^{\delta}\right), \quad W^{\delta}=W\left(n^{\delta}, T^{\delta}\right) .
$$

More precisely, we obtain from (2.8), (2.9) the relations

$$
\mu^{\delta}=T^{\delta} \ln \frac{n^{\delta}}{T^{\delta} Q^{0}\left(T^{\delta}\right)}, \quad W^{\delta}=\frac{Q^{1}\left(T^{\delta}\right)}{Q^{0}\left(T^{\delta}\right)} T^{\delta} n^{\delta} .
$$


We approximate $n^{\delta}$ and $T^{\delta}$ as follows:

$$
\begin{aligned}
n^{\delta} & =n^{0}+O(\delta), \\
T^{\delta} & =T^{0}+\delta T^{\perp}+O\left(\delta^{2}\right) \quad \text { as } \delta \rightarrow 0 .
\end{aligned}
$$

Then we can decompose $\mu^{\delta}, W^{\delta}$ and $R^{\delta}=R\left(W\left(n^{\delta}, T^{\delta}\right)\right)$ as

$$
\begin{aligned}
\mu^{\delta} & =\bar{\mu}+\delta \frac{\overline{\partial \mu}}{\partial T} T^{\perp}+O\left(\delta^{2}\right), \\
W^{\delta} & =\bar{W}+\delta \frac{\overline{\partial W}}{\partial T} T^{\perp}+O\left(\delta^{2}\right), \\
R^{\delta} & =\bar{R}+\delta \frac{\overline{\partial R}}{\partial T} T^{\perp}+O\left(\delta^{2}\right),
\end{aligned}
$$

where the overlined functions are evaluated at $\left(n^{\delta}, T^{0}\right)$, for instance

$$
\bar{\mu} \stackrel{\text { def }}{=} \mu\left(n^{\delta}, T^{0}\right), \quad \bar{W} \stackrel{\text { def }}{=} W\left(n^{\delta}, T^{0}\right), \quad \bar{R} \stackrel{\text { def }}{=} R\left(n^{\delta}, T^{0}\right) .
$$

Similarly, using Eqs. (2.16) and (2.17), we can write the current densities as follows:

$$
\begin{aligned}
j_{n}^{\delta} & =-\overline{L_{11}} \frac{E}{T^{0}}+\delta\left(-\overline{L_{11}} \nabla \frac{\bar{\mu}}{T^{0}}-\frac{\partial}{\partial T}\left(\overline{\frac{L_{11}}{T}}\right) T^{\perp} E-\overline{L_{12}} \nabla\left(-\frac{1}{T^{0}}\right)\right)+O\left(\delta^{2}\right) \\
& =\overline{j_{n}}+\delta j_{n}^{\perp}+O\left(\delta^{2}\right), \\
j_{W}^{\delta} & =-\overline{L_{21}} \frac{E}{T^{0}}+\delta\left(-\overline{L_{21}} \nabla \frac{\bar{\mu}}{T^{0}}-\frac{\partial}{\partial T}\left(\overline{\frac{L_{21}}{T}}\right) T^{\perp} E-\overline{L_{22}} \nabla\left(-\frac{1}{T^{0}}\right)\right)+O\left(\delta^{2}\right) \\
& =\overline{j_{w}}+\delta j_{w}^{\perp}+O\left(\delta^{2}\right) .
\end{aligned}
$$

Substitution of the decomposition for $j_{n}^{\delta}$ into (2.14) yields

$$
\partial_{t} n^{\delta}+\operatorname{div}\left(\overline{j_{n}}+\delta j_{n}^{\perp}\right)=O\left(\delta^{2}\right)
$$

In the similar way, we obtain for (2.15):

$$
\partial_{t} W^{\delta}+\operatorname{div} \overline{j_{W}}=\frac{1}{\delta}\left(-E \cdot \overline{j_{n}}+\bar{R}\right)-E \cdot j_{n}^{\perp}+\frac{\overline{\partial R}}{\partial T} T^{\perp}+O(\delta) .
$$

The $O\left(\delta^{-1}\right)$-term gives

$$
E \cdot \overline{j_{n}}=\bar{R}
$$

such that with the definition $\overline{j_{n}}=-\overline{L_{11}} E / T_{0}$ we get

$$
|E|^{2}=-\frac{\bar{R}}{\overline{L_{11}}} T^{0}=-\frac{R\left(n^{\delta}, T^{0}\right)}{L_{11}\left(n^{\delta}, T^{0}\right)} T^{0}
$$


Therefore, $T^{0}$ is the (unique) solution to (4.4) which exists due to (H5). Notice that in view of assumptions (H1)-(H4), the quotient $R / L_{11}$ and hence $T^{0}$ do not depend on $n^{\delta}$ (see $(2.22)$ ), i.e. $T^{0}=T(|E|)$.

From the $O(1)$-terms in (4.3) we obtain an expression for $T^{\perp}$. Indeed, substitution of the definition of $j_{n}^{\perp}$ in the $O(1)$-terms of (4.3) leads to

$$
\begin{aligned}
\partial_{t} W^{\delta}+\operatorname{div} \overline{j_{W}}= & -E \cdot j_{n}^{\perp}+\frac{\overline{\partial R}}{\partial T} T^{\perp} \\
= & \left(\frac{\partial}{\partial T}\left(\frac{\overline{L_{11}}}{T}\right)|E|^{2}+\frac{\overline{\partial R}}{\partial T}\right) T^{\perp} \\
& +E \cdot\left(\overline{L_{11}} \nabla \frac{\bar{\mu}}{T^{0}}+\overline{L_{12}} \nabla\left(-\frac{1}{T^{0}}\right)\right) .
\end{aligned}
$$

Now, the unique solvability of (4.4) with respect to $T$ implies that

$$
\frac{\partial}{\partial T}\left(\frac{\overline{L_{11}}}{T}\right)|E|^{2}+\frac{\overline{\partial R}}{\partial T}=\frac{\partial}{\partial T}\left(\frac{L_{11}}{T}|E|^{2}+R\right)\left(n^{\delta}, T^{0}\right) \neq 0 .
$$

Therefore,

$$
\Lambda \stackrel{\text { def }}{=}\left[\frac{\partial}{\partial T}\left(\frac{\overline{L_{11}}}{T}\right)|E|^{2}+\frac{\overline{\partial R}}{\partial T}\right]^{-1}
$$

is well defined and we obtain from (4.5)

$$
T^{\perp}=\Lambda\left[\frac{\partial W^{\delta}}{\partial t}+\operatorname{div} \overline{j_{W}}-E \cdot\left(\overline{L_{11}} \nabla \frac{\bar{\mu}}{T^{0}}+\overline{L_{12}} \nabla\left(-\frac{1}{T^{0}}\right)\right)\right] .
$$

The term $\partial W^{\delta} / \partial t$ equals, by the chain rule and (4.2),

$$
\left(\frac{\partial W^{\delta}}{\partial n}+\frac{\partial W^{\delta}}{\partial T} \frac{\partial T}{\partial n}\right)\left(n^{\delta}, T^{0}\right) \frac{\partial n^{\delta}}{\partial t}=-\frac{\overline{\partial W}}{\partial n} \operatorname{div} \overline{j_{n}}+O(\delta),
$$

since $T=T^{0}$ does not depend on $n^{\delta}$. Hence, we can substitute $T^{\perp}$ in $j_{n}^{\perp}$ by the above expression to obtain up to order $O(\delta)$

$$
\begin{aligned}
j_{n}^{\perp}= & -\left[1-\frac{\partial}{\partial T}\left(\frac{\overline{L_{11}}}{T}\right) \Lambda|E|^{2}\right]\left[\overline{L_{11}} \nabla \frac{\bar{\mu}}{T^{0}}+\overline{L_{12}} \nabla\left(-\frac{1}{T^{0}}\right)\right] \\
& -\frac{\partial}{\partial T}\left(\frac{\overline{L_{11}}}{T}\right) \Lambda E\left[\frac{\overline{\partial W}}{\partial n} \operatorname{div}\left(\overline{L_{11}} \frac{E}{T^{0}}\right)-\operatorname{div}\left(\overline{L_{21}} \frac{E}{T^{0}}\right)\right] .
\end{aligned}
$$

The function $j_{n}^{\perp}$ depends on $n^{\delta}, \nabla n^{\delta}, E$, and $\operatorname{div} E$. Therefore we can rewrite $(4.2)$ as

$$
\partial_{t} n^{\delta}+\operatorname{div}\left(\delta j_{n}^{\perp}\left(n^{\delta}, \nabla n^{\delta}, E, \operatorname{div} E\right)+\overline{j_{n}}\left(E, n^{\delta}\right)\right)=0,
$$

where $\overline{j_{n}}\left(E, n^{\delta}\right)$ is given by

$$
\overline{j_{n}}\left(E, n^{\delta}\right)=-L_{11}\left(n^{\delta}, T(|E|)\right) \frac{E}{T(|E|)} .
$$


Using (4.1), we can rewrite (4.6) as

$$
j_{n}^{\perp}=-D\left(n^{\delta}, E\right) \nabla n^{\delta}+F\left(n^{\delta}, E, \operatorname{div} E\right),
$$

where the diffusion matrix is defined by

$$
\begin{aligned}
D(n, E)= & \left(1-\frac{\partial}{\partial T}\left(\frac{L_{11}}{T}\right) \Lambda|E|^{2}\right) \frac{L_{11}}{n} I \\
& +\frac{\partial}{\partial T}\left(\frac{L_{11}}{T}\right) \frac{\Lambda}{T}\left(\frac{\partial W}{\partial n} \frac{\partial L_{11}}{\partial n}-\frac{\partial L_{21}}{\partial n}\right)(E \otimes E),
\end{aligned}
$$

$I$ is the unit matrix of $\mathbb{R}^{d \times d}$ and $(E \otimes E)_{i j}=E_{i} E_{j}$. The drift part $F(n, E, \operatorname{div} E)$ is given by:

$$
\begin{aligned}
F(n, E, \operatorname{div} E)= & \left(1-\frac{\partial}{\partial T}\left(\frac{L_{11}}{T}\right) \Lambda|E|^{2}\right)\left(\frac{3}{2} T L_{11}-L_{12}\right) \frac{\nabla T}{T^{2}} \\
& -\frac{\partial}{\partial T}\left(\frac{L_{11}}{T}\right) \frac{\Lambda}{T}\left(\frac{\partial W}{\partial n} \frac{\partial L_{11}}{\partial T}-\frac{\partial W}{\partial n} \frac{L_{11}}{T}+\frac{L_{21}}{T}\right)(E \otimes E) \nabla T \\
& -\frac{\partial}{\partial T}\left(\frac{L_{11}}{T}\right) \frac{\Lambda}{T}\left(\frac{\partial W}{\partial n} L_{11}-L_{21}\right) E \operatorname{div} E .
\end{aligned}
$$

Hence, the high-field drift-diffusion model for general band diagrams reads (for $\delta=1$ )

$$
\begin{aligned}
& \partial_{t} n-\operatorname{div}\left(D(n, E) \nabla n-F(n, E, \operatorname{div} E)+\frac{L_{11}(n, T(E \mid))}{T(|E|)} E\right)=0, \\
& \operatorname{div} E=n-C(x) .
\end{aligned}
$$

A necessary condition for the well-posedness of Eq. (4.8) is given by the positive definiteness of $D(n, E)$. A sufficient condition is proved in the following lemma.

Lemma 4.1 The symmetric matrix $D(n, E)$ defined in (4.7) is positive definite if for all $|E| \geq 0$,

$$
\begin{array}{r}
\frac{\partial}{\partial T}\left(\frac{L_{11}}{T}\right) \frac{\Lambda}{T}\left(\frac{\partial W}{\partial n} \frac{\partial L_{11}}{\partial n}-\frac{\partial L_{21}}{\partial n}\right) \leq 0 \\
\frac{L_{11}}{n}+\frac{\partial}{\partial T}\left(\frac{L_{11}}{T}\right)|E|^{2} \frac{\Lambda}{T}\left(-\frac{T L_{11}}{n}+\frac{\partial W}{\partial n} \frac{\partial L_{11}}{\partial n}-\frac{\partial L_{21}}{\partial n}\right)>0
\end{array}
$$

Proof. Let $\xi \in \mathbb{R}^{d}$. Then, using the inequality $|E \cdot \xi| \leq|E| \cdot|\xi|$ and the assumption (4.10),

$$
\begin{aligned}
\xi^{T} D(n, E) \xi= & \frac{L_{11}}{n}|\xi|^{2}-\frac{\partial}{\partial T}\left(\frac{L_{11}}{T}\right) \Lambda \frac{L_{11}}{n}|E|^{2}|\xi|^{2} \\
& +\frac{\partial}{\partial T}\left(\frac{L_{11}}{T}\right) \frac{\Lambda}{T}\left(\frac{\partial W}{\partial n} \frac{L_{11}}{\partial n}-\frac{\partial L_{21}}{\partial n}\right)|E \cdot \xi|^{2} \\
\geq & \delta(|E|)|\xi|^{2}
\end{aligned}
$$


with

$$
\delta(|E|)=\frac{L_{11}}{n}+\frac{\partial}{\partial T}\left(\frac{L_{11}}{T}\right)|E|^{2} \frac{\Lambda}{T}\left(-\frac{T L_{11}}{n}+\frac{\partial W}{\partial n} \frac{\partial L_{11}}{\partial n}-\frac{\partial L_{21}}{\partial n}\right) .
$$

Thus, by assumption (4.11), the assertion follows.

\subsection{Parabolic band approximation}

In the parabolic band case, it holds (see $[7,10])$ :

$$
\begin{aligned}
L_{11} & =\frac{2}{\sqrt{\pi}} \Gamma(2-\beta) d_{0} n T^{1 / 2-\beta}, \\
L_{21} & =\frac{2}{\sqrt{\pi}} \Gamma(3-\beta) d_{0} n T^{3 / 2-\beta}, \\
W & =\frac{3}{2} n T, \\
R & =\frac{2}{\sqrt{\pi}} \Gamma(2+\beta) n T^{-1 / 2+\beta} \frac{T_{0}-T}{\tau_{0}} .
\end{aligned}
$$

Lemma 4.2 Let $\beta \leq \frac{1}{2}$. Then the diffusion matrix $D(n, E)$ is uniformly positive definite for all $T \geq T_{0}$.

Proof. By Lemma 4.1, it is sufficient to prove (4.10) and (4.11). Elementary computations give

$$
\begin{aligned}
\frac{\partial}{\partial T}\left(\frac{L_{11}}{T}\right) & =-\frac{2}{\sqrt{\pi}} \Gamma(2-\beta)\left(\frac{1}{2}+\beta\right) d_{0} n T^{-3 / 2-\beta} \leq 0 \\
\frac{\partial}{\partial T} R & =\frac{2}{\sqrt{\pi}} \Gamma(2+\beta) \frac{n T^{-1 / 2+\beta}}{\tau_{0}}\left(\left(\beta-\frac{1}{2}\right) \frac{T_{0}}{T}-\left(\frac{1}{2}+\beta\right)\right) \leq 0
\end{aligned}
$$

and hence

$$
\frac{\partial}{\partial T}\left(\frac{L_{11}}{T}\right) \Lambda=\frac{\frac{\partial}{\partial T}\left(L_{11} / T\right)|E|^{2}}{\frac{\partial}{\partial T}\left(L_{11} / T\right)|E|^{2}+\frac{\partial}{\partial T} R}|E|^{-2}>0
$$

Furthermore, it holds

$$
\frac{\partial W}{\partial n} \frac{\partial L_{11}}{\partial n}-\frac{\partial L_{21}}{\partial n}=\frac{2}{\sqrt{\pi}} \Gamma(2-\beta)\left(\beta-\frac{1}{2}\right) d_{0} T^{3 / 2-\beta} \leq 0,
$$

if $\beta \leq \frac{1}{2}$. This shows that the condition (4.10) is satisfied. It remains to estimate $\delta(|E|)$ defined in (4.12). Using

$$
\frac{\partial}{\partial T}\left(\frac{L_{11}}{T}\right)|E|^{2} \Lambda
$$




$$
\begin{aligned}
& =\frac{\left(\frac{1}{2}+\beta\right) \Gamma(2-\beta) d_{0}|E|^{2}}{\left(\frac{1}{2}+\beta\right) \Gamma(2-\beta) d_{0}|E|^{2}+\Gamma(2+\beta) \tau_{0}^{-1}\left(\left(\frac{1}{2}-\beta\right) T_{0}+\left(\frac{1}{2}+\beta\right) T\right) T^{2 \beta}} \\
& =\frac{\left(\frac{1}{2}+\beta\right)\left(T-T_{0}\right)}{\left(\frac{1}{2}+\beta\right)\left(T-T_{0}\right)+\left(\left(\frac{1}{2}-\beta\right) T_{0}+\left(\frac{1}{2}+\beta\right) T\right)} \\
& =\frac{\left(\frac{1}{2}+\beta\right)\left(T-T_{0}\right)}{(1+2 \beta) T-2 \beta T_{0}}
\end{aligned}
$$

which follows from (3.8), and

$$
-\frac{T L_{11}}{n}+\frac{\partial W}{\partial n} \frac{\partial L_{11}}{\partial n}-\frac{\partial L_{21}}{\partial n}=\frac{2}{\sqrt{\pi}} \Gamma(2-\beta)\left(-\frac{3}{2}+\beta\right) d_{0} T^{3 / 2-\beta},
$$

we obtain for $T \geq T_{0}$,

$$
\begin{aligned}
\delta(|E|)= & \frac{2}{\sqrt{\pi}} \Gamma(2-\beta) d_{0} T^{1 / 2-\beta} \\
& +\frac{(1+2 \beta)\left(T-T_{0}\right)}{(1+2 \beta) T-2 \beta T_{0}} \frac{1}{\sqrt{\pi}} \Gamma(2-\beta)\left(-\frac{3}{2}+\beta\right) d_{0} T^{1 / 2-\beta} \\
= & \frac{1}{\sqrt{\pi}} \Gamma(2-\beta) d_{0} T^{1 / 2-\beta}\left(2-\left(\frac{3}{2}-\beta\right) \frac{(1+2 \beta) T-(1+2 \beta) T_{0}}{(1+2 \beta) T-2 \beta T_{0}}\right) \\
\geq & \frac{1}{\sqrt{\pi}} \Gamma(2-\beta) d_{0} T^{1 / 2-\beta}\left(2-\left(\frac{3}{2}-\beta\right)\right) \\
= & \frac{d_{0}}{\sqrt{\pi}} \Gamma(2-\beta)\left(\frac{1}{2}+\beta\right) T^{1 / 2-\beta} \\
\geq & \frac{d_{0}}{\sqrt{\pi}} \Gamma(2-\beta)\left(\frac{1}{2}+\beta\right) T_{0}^{1 / 2-\beta} \\
> & 0
\end{aligned}
$$

using $\beta \leq \frac{1}{2}$ again.

With the use of Eqs. (4.13) and (3.8) we obtain, after elementary but lengthy computations, the following expressions:

$$
\begin{aligned}
& \begin{array}{l}
D(n, E) \\
=\frac{2}{\sqrt{\pi}} \frac{\Gamma(2-\beta) d_{0} T^{1 / 2-\beta}}{T+2 \beta\left(T-T_{0}\right)}\left[\left(\left(\frac{1}{2}+\beta\right) T+\left(\frac{1}{2}-\beta\right) T_{0}\right) I\right. \\
\left.\quad-\frac{\left(\frac{1}{2}+\beta\right)\left(\frac{1}{2}-\beta\right) d_{0} \tau_{0}}{\Gamma(2+\beta) T^{2 \beta}}(E \otimes E)\right], \\
F(n, E, \operatorname{div} E) \\
=\frac{2}{\sqrt{\pi}} \frac{\Gamma(2-\beta)\left(\frac{1}{2}-\beta\right) d_{0} n T^{3 / 2-\beta}}{(1+2 \beta) T-2 \beta T_{0}}\left[-\left(\left(\frac{1}{2}+\beta\right) T+\left(\frac{1}{2}-\beta\right) T_{0}\right) \frac{\nabla T}{T^{2}}\right. \\
\left.\quad-\frac{5\left(\frac{1}{2}+\beta\right) \Gamma(2-\beta) d_{0} \tau_{0}}{2 \Gamma(2+\beta) T^{2(\beta+1)}}(E \otimes E) \nabla T+\frac{\left(\frac{1}{2}+\beta\right) \Gamma(2-\beta) d_{0} \tau_{0}}{\Gamma(2+\beta) T^{2(\beta+1)}}(\operatorname{div} E) E\right] .
\end{array}
\end{aligned}
$$


In the Chen case $\beta=\frac{1}{2}$ the above formulas simplify to

$$
\begin{aligned}
D(n, E) & =\frac{d_{0}}{2-T_{0} / T(|E|)}, \\
F(n, E, \operatorname{div} E) & =0 .
\end{aligned}
$$

Therefore, the high-field drift-diffusion model for parabolic bands in the Chen case reads (see (4.8)):

$$
\partial_{t} n-\operatorname{div}\left(\frac{d_{0}}{2-T_{0} / T(|E|)} \nabla n+\frac{n}{T(E \mid)} E\right)=0,
$$

and $T(|E|)$ is given by (3.10). Notice that for $|E| \rightarrow \infty$, the diffusivity converges to $d_{0} / 2$ :

$$
\frac{d_{0}}{2-T_{0} / T(|E|)} \rightarrow \frac{d_{0}}{2} \quad \text { as }|E| \rightarrow \infty
$$

whereas for $|E| \rightarrow 0$, we obtain:

$$
\frac{d_{0}}{2-T_{0} / T(|E|)} \rightarrow d_{0} \quad \text { as }|E| \rightarrow 0
$$

For the drift term it holds

$$
\frac{n}{T(|E|)} E \sim \frac{3}{8 d_{0} \tau_{0}} \frac{E}{|E|} \quad \text { as }|E| \rightarrow \infty
$$

\section{References}

[1] N. Ben Abdallah and P. Degond. On a hierarchy of macroscopic models for semiconductors. J. Math. Phys., 37:3308-3333, 1996.

[2] N. Ben Abdallah, P. Degond, and S. Génieys. An energy-transport model for semiconductors derived from the Boltzmann equation. J. Stat. Phys., 84:205-231, 1996.

[3] N. Ben Abdallah, P. Degond, P. Markowich, and C. Schmeiser. High field approximations of the spherical harmonics expansion model for semiconductors. To appear in Z. Angew. Math. Phys., 2000.

[4] F. Brezzi, L. Marini, and P. Pietra. Two-dimensional exponential fitting and applications to drift-diffusion models. SIAM J. Num. Anal., 26:1342-1355, 1989.

[5] D. Chen, E. Kan, U. Ravaioli, C. Shu, and R. Dutton. An improved energy transport model including nonparabolicity and non-Maxwellian distribution effects. IEEE Electr. Dev. Letters, 13:26-28, 1992. 
[6] P. Degond, S. Génieys, and A. Jüngel. A system of parabolic equations in nonequilibrium thermodynamics including thermal and electrical effects. J. Math. Pures Appl., 76:991-1015, 1997.

[7] P. Degond, A. Jüngel, and P. Pietra. Numerical discretization of energy-transport model for semiconductors with non-parabolic band structure. To appear in SIAM J. Sci. Comp., 2000.

[8] P. Dmitruk, A. Paul, and L. Reyna. High electric field approximation to charge transport in semiconductor devices. Appl. Math. Lett., 5:99-102, 1992.

[9] C. Jacoboni and P. Lugli. The Monte Carlo Method for Semiconductor Device Simulation. Springer, Wien, 1989.

[10] A. Jüngel. Quasi-hydrodynamic Semiconductor Equations. To appear in Progress in Nonlinear Differential Equations and Its Applications. Birkhäuser, Basel, 2000.

[11] E. Kane. J. Phys. Chem. Solids, 1:249, 1957.

[12] E. Lyumkis, B. Polsky, A. Shur, and P. Visocky. Transient semiconductor device simulation including energy balance equation. Compel, 11:311-325, 1992.

[13] F. Poupaud. Runaway phenomena and fluid approximation under high fields in semiconductor kinetic theory. Z. Angew. Math. Mech., 72:359-372, 1992.

[14] C. Schmeiser and A. Zwirchmayr. Elastic and drift-diffusion limits of electronphonon interaction in semiconductors. Math. Models Meth. Appl. Sci., 8:37-53, 1998.

[15] S. Selberherr. Analysis and Simulation of Semiconductor Devices. Springer, Wien, 1984.

[16] R. Stratton. Diffusion of hot and cold electrons in semiconductor barriers. Phys. Rev., 126:2002-2014, 1962.

[17] D. Ventura, A. Gnudi, G. Baccarani, and F. Odeh. Multidimensional spherical harmonics expansion of Boltzmann equation for transport in semiconductors. Appl. Math. Lett., 5:85-90, 1992. 